\title{
Manejo de la neumonía comunitaria del adulto mayor en el ámbito ambulatorio
}

\author{
Philippa Moore ${ }^{1}$, Juan Pablo O rtega², Fernando Saldías ${ }^{3}$, \\ María Angélica 0 yarzúna.
}

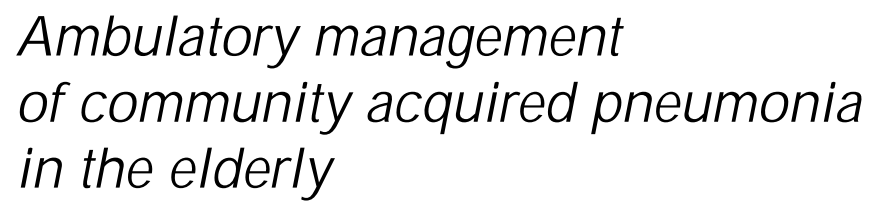

Community Acquired Pneumonia (CAP) is the first cause of death by respiratory disease in Chile and the first specific cause of death in people over 80 years of age. The geriatric population has a greater risk of suffering pneumonia, its complications and consequently dying. This is not only related to chronological age but also to certain factors related to ageing such as the presence of comorbidity, malnutrition, and cognitive impairment. An atypical presentation that delays the diagnosis and treatment also increases the risk of complications. CAP in the elderly is caused by the same pathogens that cause it in younger patients. Spneumoniae is the main pathogen followed by viral infections particularly in winter. An important strategy to reduce CAP related health costs, is the identification of patients who are at low risk of complications and who therefore could be managed at home. Optimum management of CAP in the elderly includes early diagnosis and the definition of clinical severity, early antibiotic treatment at the right dose and for an adequate length of time and a correct decision whether the patient should be managed in hospital or at home (Rev Méd Chile 2006; 134: 1568-75).

(Key words: Aged, 80 and over; Pneumonia; Streptococcus pneumoniae)

\footnotetext{
Recibido el 27 de marzo, 2006. Aceptado el 14 de junio, 2006.

${ }^{1}$ Departamento Medicina Familiar, Pontificia Universidad Católica de Chile. ${ }^{2}$ Departamento Medicina Interna, Hospital Sótero del Río. ${ }^{3}$ Departamento de Enfermedades Respiratorias, Pontificia Universidad Católica de Chile.

anterna de Medicina, Pontificia Universidad Católica de Chile.
}

L as enfermedades respiratorias constituyen la tercera causa de muerte de la población chilena $^{1,2}$. El 50\% de los decesos por enfermedades respiratorias en el adulto son atribuibles a

Correspondencia a: Dra. Philippa Moore. Departamento de Medicina Familiar. Lira 44, Santiago, Chile. Fax: 518 6760. E mail: moore@med.puc.cl neumonía. La neumonía es la principal causa de muerte por enfermedades infecciosas en la población chilena y la primera causa específica de muerte en los mayores de 80 años ${ }^{1,2}$. La mortalidad en adultos entre 20 y 64 años es relativamente baja (14,8 por 100.000 habitantes), siendo el riesgo de muerte 40 veces superior en la población senescente. La neumonía adquirida en la comunidad (NAC) continúa siendo una de las 
principales causas de consulta ambulatoria, hospitalización y muerte en el adulto mayor ( $>65$ años) de los países desarrollados y en vías de desarrollo, constituyéndose en un importante problema de salud pública a nivel mundial ${ }^{3}$.

La población senescente en nuestro país ha aumentado en los últimos años, correspondiendo a $8 \%$ de la población según el censo del año $2002^{4}$. Se estima que en la próxima década, Chile incrementará significativamente su población de adultos mayores.

La incidencia anual estimada de NAC en el adulto mayor, no institucionalizado, fluctúa entre 25 y 44 por 1.000 , siendo cuatro veces mayor que en el adulto joven ${ }^{5}$. Considerando que la neumonía comunitaria no es una condición sujeta a vigilancia epidemiológica, la estimación de su incidencia es incierta y se sustenta en la información disponible en el ambiente hospitalario. Así, se registraron 78.661 egresos hospitalarios por neumonía en 2001, correspondiendo a 43,6\% de los egresos por enfermedades respiratorias en los sistemas público y privado de salud ${ }^{1}$.

Se ha comunicado una mayor prevalencia, riesgo de complicaciones y muerte en la NAC del adulto mayor. Además, esta infección se ha reconocido como un marcador de mal pronóstico en la evolución a corto plazo ${ }^{6}$. Sin embargo, la edad avanzada por sí sola probablemente no constituya el único factor determinante del mal pronóstico en la neumonía del anciano. Factores de gravedad aun mal definidos en esta población, tales como la presencia de comorbilidades múltiples (cardiopatías, EPOC, diabetes, insuficiencia renal crónica, enfermedades neurológicas, neoplasias), malnutrición y demencia; sumado al cuadro clínico atípico u oligosintomático, que se han asociado a un retraso en el diagnóstico e inicio del tratamiento antimicrobiano; pueden afectar adversamente la evolución y pronóstico del paciente senescente con neumonía ${ }^{7-9}$.

\section{FACTORES DE RIESGO}

En un estudio comunitario finlandés, que incluyó 4.175 adultos mayores de 60 años ${ }^{10}$, se identificaron los siguientes factores de riesgo de adquisición de neumonía: alcoholismo (RR: 9,0; IC 95\%: 5,1-16,2), asma bronquial (RR: 4,2; IC 95\%: 3,35,4), inmunosupresión (RR: 3,1; IC 95\%: 1,9-5,1), enfermedad pulmonar crónica (RR: 3,0; IC 95\%: 2,3-3,9), cardiopatías (RR: 1,9; IC 95\%: 1,7-2,3), institucionalización (RR: 1,8; IC 95\%: 1,4-2,4) y edad avanzada ( $\geq 70$ años versus $60-69$ años; RR: 1,5; IC 95\%: 1,3-1,7). Varios estudios han destacado la importancia de la edad, presencia de enfermedades crónicas y los trastornos de la deglución como factores de riesgo independiente de adquirir una infección pulmonar.

La edad como factor de riesgo. Asociado al envejecimiento, se producen una serie de cambios en la fisiología del sistema respiratorio, que favorecen las infecciones respiratorias y determinan su mayor gravedad. Los cambios fisiológicos más importantes son: a) Disminución de la fuerza y tolerancia a la fatiga, de los músculos respiratorios, secundario a la pérdida de sarcómeros, malnutrición y sobrecarga de los músculos inspiratorios ${ }^{5}$; b) Disminución en el soporte elástico pulmonar, que se traduce en un aumento de la capacidad residual funcional, que conlleva a un aumento del esfuerzo respiratorio; c) Disminución de la distensibilidad de la caja torácica, secundario a calcificaciones de las articulaciones costales y a fracturas vertebrales por aplastamiento, que ocasionan aumento de la cifosis dorsal y diámetro anteroposterior del tórax, lo cual disminuye la curvatura del diafragma reduciendo su capacidad de generar fuerza y tensión ${ }^{11}$. Todos estos cambios provocan una alteración de la mecánica respiratoria que se traduce en una disminución de los flujos espiratorios y la efectividad de la tos, lo que impide una adecuada eliminación de las secreciones bronquiales.

La respuesta inmune celular mediada por linfocitos $\mathrm{T}$ y la capacidad de producción de anticuerpos están disminuidas en el senescente ${ }^{12}$. Los cambios en la respuesta inmune del anciano, asociado a un aumento en el riesgo de broncoaspiración por deterioro de los mecanismos de defensa de la vía aérea, disminución del clearance mucociliar y la presencia de enfermedades crónicas, que favorecen la aspiración (demencia, trastornos de la deglución y accidentes vasculares encefálicos); explican la mayor incidencia y letalidad de la infección pulmonar en esta población.

Etiología. Idealmente, el tratamiento antibiótico debiera ser dirigido contra el agente causal de la 
infección. Sin embargo, en la mayoría de los casos de neumonía, las limitaciones de disponibilidad, sensibilidad y especificidad, de las técnicas de diagnóstico microbiológico, obligan a iniciar un tratamiento empírico, el cual debe estar basado en estudios epidemiológicos que permitan presumir el agente causal de la infección en un paciente determinado.

La identificación del agente causal de la neumonía en el adulto mayor constituye un desafío, puesto que más de $50 \%$ de los pacientes no presenta expectoración al efectuarse el diagnóstico. En general, la neumonía del anciano es causada por los mismos patógenos que en el adulto joven $8,13,14$. Los principales microorganismos involucrados en la neumonía del anciano son: S pneumoniae, $\mathrm{H}$ influenzae, bacilos gram negativos entéricos, S aureus y los virus respiratorios (influenza, parainfluenza y sincicial respiratorio) (Tabla 1$)^{5}$. La infección por microorganismos atípicos ( $\mathrm{M}$ pneumoniae, $\mathrm{C}$ pneumoniae y $\mathrm{C}$ psittacci) es menos prevalente en esta población, que en el adulto joven (OR: 2,3; IC 95\%: 1,2$4,5)^{14}$. En un estudio descriptivo nacional que involucró 463 adultos inmunocompetentes hospitalizados por NAC, se logró identificar el agente causal en cerca de $25 \%$ de los casos $^{15}$. El S pneumoniae fue el principal agente causal de neumonía (10\%), seguido por los bacilos gram negativos entéricos (5\%), S aureus (4\%) y $\mathrm{H}$ influenzae (4\%).

Las infecciones respiratorias de origen viral adquieren importancia durante los meses de invierno, especialmente entre los ancianos institucionalizados. Entre los pacientes que se hospitalizan por esta causa, los principales agentes involucrados son los virus influenza A, sincicial respiratorio y parainfluenza. En nuestro medio, Díaz y cols han destacado la importancia de los virus respiratorios en el adulto hospitalizado por neumonía comunitaria ${ }^{16}$.

Los estudios etiológicos realizados en nuestro medio han involucrado pacientes hospitalizados, no existiendo datos nacionales sobre la etiología de la neumonía que se maneja en el medio ambulatorio. Es importante destacar que el cuadro clínico y los hallazgos de la radiografía de tórax no permiten predecir el agente causal.

Diagnóstico clínico-radiográfico

a) Presentación clínica. El cuadro clínico del paciente con NAC puede variar entre un cuadro infeccioso leve de bajo riesgo de complicaciones, hasta uno de extrema gravedad con riesgo vital. En el adulto mayor, la presentación suele ser menos sintomática. La tríada clásica de tos, fiebre y disnea, se presenta en menos de $60 \%$ de los enfermos, mientras que $10 \%$ no refiere

Tabla 1. Etiología de la neumonía del adulto mayor, adquirida en la comunidad y en centros geriátricos 5

\begin{tabular}{|lcc|}
\hline Microorganismos & $\begin{array}{c}\text { Neumonía adquirida } \\
\text { en la comunidad }\end{array}$ & $\begin{array}{c}\text { Neumonía adquirida } \\
\text { en centro geriátrico }\end{array}$ \\
\hline Streptococcus pneumoniae & $5-58 \%$ & $4-30 \%$ \\
Haemophilus influenzae & $2-14 \%$ & $0-2 \%$ \\
Staphylococcus aureus & $0-7 \%$ & $0-4 \%$ \\
Moraxella catarrhalis & $0-4 \%$ & $2-3 \%$ \\
Escherichia coli & $1-7 \%$ & $0-2 \%$ \\
Pseudomonas aeruginosa & $1-5 \%$ & $0-4 \%$ \\
Klebsiella pneumoniae & $0-4 \%$ & $4-6 \%$ \\
Gérmenes atípicos & & $0-18 \%$ \\
Chlamydia pneumoniae & $0-28 \%$ & $1-2 \%$ \\
Mycoplasma pneumoniae & $1-13 \%$ & $0-1 \%$ \\
Legionella pneumophila & $0-15 \%$ & $0-4 \%$ \\
Virus respiratorios & & $1-2 \%$ \\
Influenza A & $1-32 \%$ & $0-4 \%$ \\
Parainfluenza & & \\
\hline
\end{tabular}


ninguno de estos síntomas. No es infrecuente que se presenten con síntomas inespecíficos como deterioro cognitivo, incontinencia urinaria, trastornos de la marcha, caídas y descompensación de enfermedades crónicas ${ }^{17}$. La taquicardia y la taquipnea están presentes en dos tercios de los ancianos con neumonía y pueden preceder a otros hallazgos clínicos hasta por 3 ó 4 días ${ }^{18}$.

b) Estudio radiológico. En lo posible, la radiografía de tórax debe ser uno de los exámenes de rutina solicitados para el diagnóstico y evaluación de los pacientes con NAC. Permite confirmar el diagnóstico clínico y establecer su localización, extensión y eventuales complicaciones, además permite diferenciar la neumonía de otras patologías, y puede ser útil en el seguimiento de los pacientes de alto riesgo. En el ámbito ambulatorio, se recomienda solicitar una radiografía de tórax a los pacientes con cuadro clínico sugerente de neumonía para certificar el diagnóstico y evaluar la gravedad del cuadro, además se sugiere un control radiográfico entre las 6 y 8 semanas de finalizado el tratamiento, para evaluar patologías concomitantes que pueden estar facilitando la infección o modificando su evolución, como procesos obstructivos bronquiales, enfisema pulmonar, bronquiectasias, etc.

c) Exámenes de laboratorio. En general, no se recomienda realizar estudios microbiológicos ni bioquímicos de rutina en los pacientes manejados ambulatoriamente considerando su buen pronóstico y bajo riesgo de complicaciones (letalidad inferior a 1\%-2\%). En nuestro país, se recomienda obtener muestras de expectoración para baciloscopias y cultivo de Koch en pacientes con tos persistente y compromiso de su estado general.

d) Diagnóstico diferencial. Debe plantearse en aquellos pacientes con cuadro clínico sugerente de NAC, en quienes una adecuada terapia antibiótica, no se traduce en mejonía clínica. En el diagnóstico diferencial se debe incluir la tuberculosis y la infección por patógenos inhabituales (Nocardia asteroides, Aspergillus sp, Mycobacterium avium), el cáncer bronquial y los trastomos inflamatorios no infecciosos, como la neumonitis por hipersensibilidad, daño pulmonar por drogas, neumonía en organización criptogénica y enfermedades del colágeno, entre otras.
Evaluación de la gravedad. El paciente con neumonía que no presenta factores de riesgo, manejado ambulatoriamente tiene una letalidad inferior a $1 \%-2 \%$, elevándose a $5 \%-15 \%$ en los pacientes con comorbilidad o factores de riesgo específicos que son admitidos al hospital; ascendiendo a $20 \%-50 \%$ en pacientes con NAC grave admitidos a la Unidad de Cuidados Intensivos 7,8,15.

Factores de riesgo de evolución complicada y muerte, en la neumonía del anciano son la edad avanzada (OR: 1,8; IC 95\%: 1,1-3,1), presencia de comorbilidad (OR: 4,1; IC 95\%: 2,1-8,1), trastornos de la respuesta motora (OR: 2,3; IC 95\%: 1,4-3,7), signos vitales anormales (OR: 3,4; IC 95\%: 2,1-5,4) y falla renal aguda (OR: 2,5; IC 95\%: 1,5-4,2) ${ }^{19}$.

La identificación de los pacientes de bajo riesgo de complicaciones, susceptibles de ser manejados en el medio ambulatorio, permitiría reducir significativamente los costos de la atención de salud, minimizando los riesgos asociados, sin comprometer la evolución y pronóstico de los enfermos. Con este propósito las guías clínicas internacionales han recomendado utilizar el Índice de Gravedad de la Neumonía descrito por Fine y cols, para la pesquisa de pacientes de bajo riesgo, y el Índice de Gravedad de la Sociedad Británica de Tórax para la pesquisa de pacientes de alto riesgo con neumonía grave, quienes deben ser manejados en la $\mathrm{UCI}^{20}$. Ambos índices han sido validados en adultos hospitalizados por NAC en el medio nacional ${ }^{8,15}$. Sin embargo, su aplicación en el ámbito ambulatorio ha sido limitada, ya que requieren de exámenes de laboratorio que no se encuentran ampliamente disponibles en los servicios de atención primaria.

En el medio nacional, se ha diseñado un instrumento pronóstico para evaluar la gravedad de los pacientes con NAC atendidos en los servicios de urgencia y consultorios de atención primaria $^{21}$. Esta herramienta incluye sólo variables clínico-radiográficas y no requiere exámenes de laboratorio complementarios como los índices pronósticos de Fine y la Sociedad Británica de Tórax (Tabla 2) ${ }^{21}$. Estos índices permiten identificar de manera sencilla a los pacientes de bajo riesgo que pueden ser tratados eficazmente en el medio ambulatorio; aquellos de riesgo intermedio que requieren una hospitalización abreviada 0 control ambulatorio estricto, y aquellos de riesgo elevado que deben ser referidos al hospital. 
Tabla 2. Índice de gravedad asociado a evolución clínica complicada y mortalidad elevada en adultos inmunocompetentes hospitalizados por neumonía comunitaria ${ }^{21}$

\begin{tabular}{|lr|}
\hline Factores de riesgo & Puntaje \\
\hline Comorbilidad & 3 \\
Compromiso de conciencia & 2 \\
P arterial sistólica $<90 \mathrm{mmHg}$ & 3 \\
F respiratoria $20-35 \mathrm{resp} / \mathrm{min}$ & 3 \\
F respiratoria $>35 \mathrm{resp} / \mathrm{min}$ & 4 \\
Temperatura $<37,5^{\circ} \mathrm{C}$ & 2 \\
Rango & $0-14$ \\
Categorías de riesgo & Letalidad a 30 días \\
\hline Categoría I (0-5 puntos) & $0,9 \%$ \\
Categoría II (6-7 puntos) & $4,9 \%$ \\
Categoría III (8-9 puntos) & $14,2 \%$ \\
Categoría IV ( $\geq 10$ puntos) & $35,6 \%$ \\
\hline
\end{tabular}

En el medio ambulatorio, la Sociedad Chilena de Enfermedades Respiratorias ha recomendado evaluar la gravedad de los pacientes con neumonía considerando las siguientes variables clínicas: edad mayor de 65 años, presencia de comorbilidad, compromiso de conciencia, alteración de los signos vitales ( $\mathrm{FC} \geq 120 \mathrm{lat} / \mathrm{min}, \mathrm{PA}<90 / 60 \mathrm{mmHg}, \mathrm{FR} \geq 20$ resp/min), compromiso radiográfico multilobar 0 bilateral, presencia de cavitación o derrame pleural, presencia de comorbilidad descompensada, y si dispone de oximetría de pulso: $\mathrm{SaO}_{2}<90 \%$ respirando aire ambiente (Figura 1).

Es importante destacar que los criterios de gravedad y los índices pronósticos no son lo suficientemente sensibles y específicos para predecir la evolución del paciente individual. Así, el juicio clínico y la experiencia del médico deben predominar sobre los modelos predictivos, los cuales no son infalibles.

\section{TRATAMIENTO}

Como se mencionó, los pacientes seleccionados apropiadamente para manejo ambulatorio tienen bajo riesgo de complicaciones y muerte. En esta categoría se reconoce a dos grupos de pacientes: a)

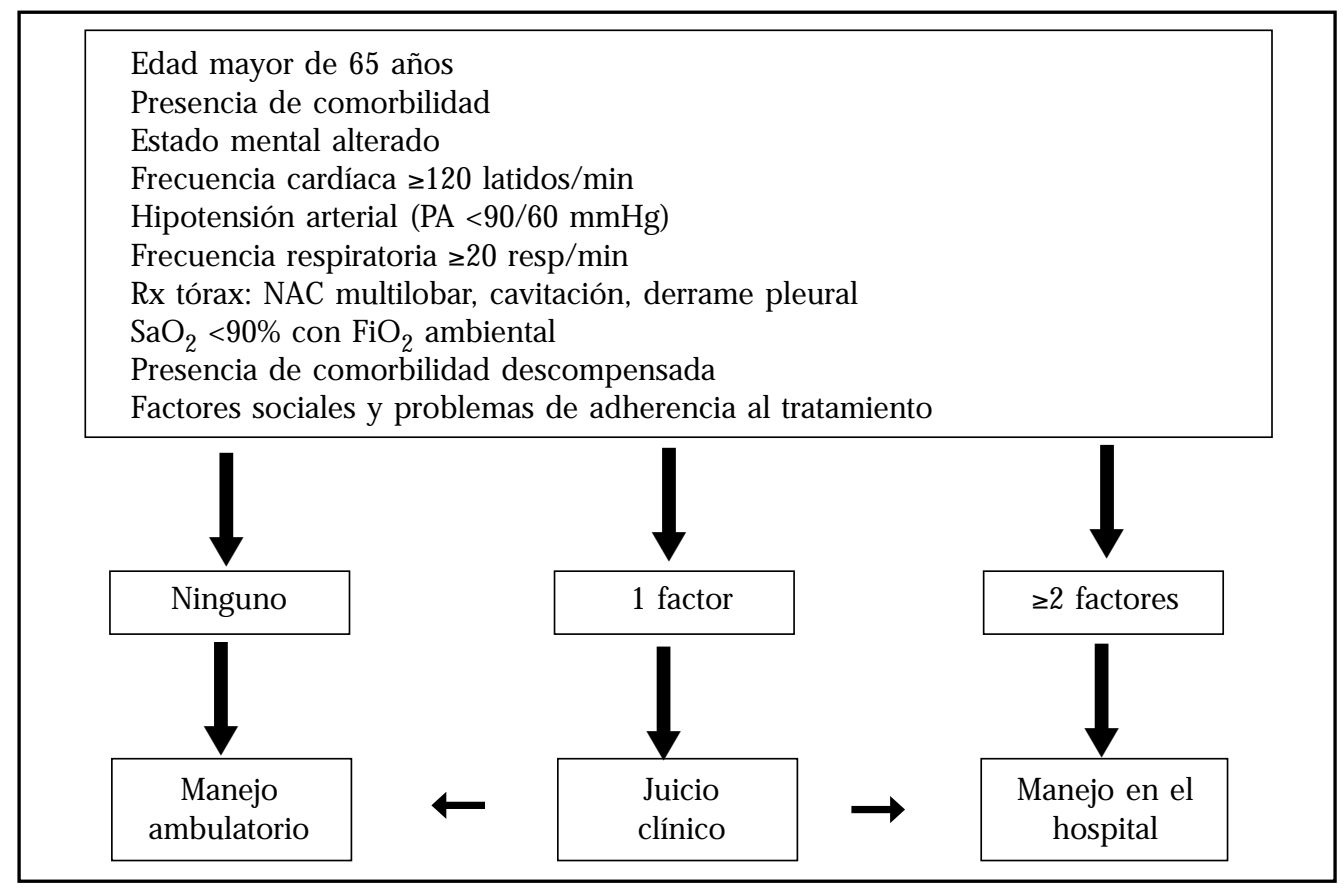

Figura 1. Recomendaciones para la evaluación de la gravedad del paciente con neumonía comunitaria atendido en el medio ambulatorio. 
Grupo 1: adultos menores de 65 años sin comorbilidad y, b) Grupo 2: adultos mayores con o sin comorbilidad asociada.

1. Medidas generales. Existe escasa evidencia sobre la efectividad de las medidas generales en el manejo ambulatorio de la NAC. Sin embargo, se suelen recomendar las siguientes indicaciones:

- Reposo en cama mientras tiene fiebre o acentuado compromiso del estado general.

- Sentarse regularmente, realizar inspiraciones profundas y promover la movilización precoz.

- Régimen blando, liviano e hidratación oral adecuada.

- Evitar el consumo de alcohol, tabaco e irritantes digestivos.

- Control periódico de la temperatura corporal.

- Considerar suplementos nutricionales si la enfermedad es prolongada.

- El dolor pleurítico puede ser manejado con paracetamol.

2. Recomendaciones de tratamiento antibiótico en el adulto mayor con neumonía, manejado en el ámbito ambulatorio. El antibiótico prescrito debe ser administrado precozmente, idealmente dentro de las $8 \mathrm{~h}$ de realizado el diagnóstico. Existe una amplia variedad de antibióticos que son eficaces en el manejo ambulatorio de pacientes con NAC y esta respuesta parece ser independiente del agente etiológico. La eficacia clínica de la amoxicilina oral en dosis mayor de $2 \mathrm{~g} /$ día, los agentes ß-lactámicos asociados a un inhibidor de la betalactamasa, los macrólidos y azálidos (eritromicina, claritromicina y azitromicina) y las cefalosporinas de $2^{2}$ generación (cefuroxima), ha sido ampliamente demostrada en varios estudios clínicos que incluyen pacientes ambulatorios y hospitalizados ${ }^{22-26}$. Además, dos metaanálisis recientes han confirmado que el tratamiento ambulatorio de la neumonía comunitaria con agentes ßs-lactámicos, macrólidos y fluoroquinolonas tienen eficacia similar ${ }^{26,27}$.

La resistencia a antibióticos de Streptococcus pneumoniae ha ido en aumento en el mundo. En un estudio reciente, el patrón de resistencia de las cepas de Spneumoniae aisladas de muestras invasivas y no invasivas en un hospital docente de Santiago mostró susceptibilidad disminuida a penicilina en $31 \%$ de los casos, a macrólidos en $21 \%$ y a cefotaxima en $16 \%{ }^{28}$. Sin embargo, ha resultado controvertido comprobar si la disminución de la susceptibilidad a los antibióticos afecta la morbimortalidad de la neumonía neumocócica $^{29,30}$. Así, varios estudios extranjeros han demostrado la eficacia de los agentes ß-lactámicos en el tratamiento de la neumonía neumocócica con CIM para penicilina inferior a $4 \mu \mathrm{g} / \mathrm{ml}$.

Basados en estos antecedentes, se recomienda que el tratamiento antibiótico empírico de la NAC deba cubrir fundamentalmente a $\mathrm{S}$ pneumoniae, $\mathrm{H}$ influenzae y ocasionalmente sea necesario cubrir los llamados microorganismos atípicos (Mycoplasma, Chlamydia y Legionella). En el adulto mayor se recomienda el uso de amoxicilina-ácido clavulánico o cefuroxima. No existen fundamentos clíni$\cos$ ni bacteriológicos que justifiquen la prescripción de tratamiento antibiótico combinado (agentes ß-lactámicos asociado a macrólidos 0 fluoroquinolonas) en el manejo de la $\mathrm{NAC}^{26,27}$. Además de los agentes beta-lactámicos (amoxicilina, amoxicilina-ácido clavulánico, cefuroxima), las fluoroquinolonas (levofloxacina, moxifloxacina y gatifloxacina), los nuevos macrólidos (claritromicina, roxitromicina) y ketólidos (telitromicina) tienen buena cobertura frente a los principales patógenos respiratorios aislados en la neumonía comunitaria del adulto mayor (S pneumoniae, $\mathrm{H}$ influenzae, $\mathrm{M}$ catarrhalis, $\mathrm{M}$ pneumoniae, $\mathrm{C}$ pneumoniae y $\mathrm{L}$ pneumophila) ${ }^{31}$. Sin embargo, se recomienda no emplear estos antimicrobianos en forma rutinaria en el ámbito ambulatorio, con el propósito de disminuir la emergencia de microorganismos resistentes a estos antimicrobianos en la comunidad.

La fisioterapia no ha demostrado mejorar la evolución y pronóstico en el manejo de los enfermos con $\mathrm{NAC}^{32}$, por lo tanto su indicación quedará a criterio del clínico.

¿Cuál es la duración del tratamiento antibiótico en el adulto mayor con neumonía manejado en el ambiente ambulatorio? No existen estudios que hayan examinado dirigidamente este tópico, comparando diferentes esquemas de tratamiento en la NAC manejada en forma ambulatoria. Las guías clínicas de la Sociedad Americana de Tórax y la Sociedad Chilena de Enfermedades Respiratorias recomiendan que el tratamiento dure entre 7-10 días (con la excepción de azitromicina que se puede administrar durante 5 días).

¿Cuándo debo controlar el paciente con neumonía manejado ambulatoriamente? El seguimiento del paciente con neumonía en el medio ambulatorio 
depende del juicio clínico y los factores de riesgo, en general se recomienda controlar el paciente:

a) A las 48-72 $\mathrm{h}$ para reevaluar los criterios de gravedad (factores pronósticos). Si no hay signos de mejoría se debe referir al hospital.

b) A los 7-10 días de tratamiento para evaluar la necesidad o no de prolongar el tratamiento.

c) Solicitar radiografía de tórax de control a las 68 semanas de seguimiento.

\section{MEDIDAS PREVENTIVAS}

Vacuna antineumocócica. Butler y cols. demostraron que la vacuna cubre cerca de $90 \%$ de las cepas que ocasionan enfermedad neumocócica invasiva (ENI) en sujetos inmunocompetentes mayores de 5 años ${ }^{33}$.

Un metaanálisis reciente demuestra que la vacuna otorga protección frente al desarmollo de ENI (OR 0,47; IC 95\%: 0,37-0,59) pero no reduce el riesgo de neumonía, la letalidad por neumonía, ni la mortalidad general. En el análisis de subgrupos, se confirma la protección contra la ENI en sujetos inmunocompetentes (OR 0,44; IC 95\%: 0,34-0,58) y en adultos mayores inmunocompetentes (OR 0,30; IC 95\%: 0,14-0,63).

Se recomienda administrar la vacuna antineumocócica a adultos mayores de 65 años, pacientes de cualquier edad portadores de enfermedades

\section{REFERENCIAS}

1. Ministerio de Salud de Chile. Departamento de Estadísticas e Información de Salud (http.// dies.minsal.cl/index.asp).

2. Szot J. Mortalidad por enfermedades respiratorias en Chile durante 1999. Rev Chil Enf Respir 2003; 19: 8-14.

3. BartLetT JG, Mundy LM. Community-acquired pneumonia. N Engl J Med 1995; 333: 1618-24.

4. Instituto Nacional de Estadísticas. Anuarios de Estadísticas Vitales, Chile, 2000 (http://www.ine.cl/ cd2002/index.php).

5. Janssens JP, Krause KH. Pneumonia in the very old. Lancet Infect Dis 2004; 4: 112-24.

6. Kaplan V, Ciermont G, Griffin MF, Kasal J, Watson RS, LINDE-Zwirble WT et al. Pneumonia: still the old man's friend? Arch Intern Med 2003; 163: 317-23. crónicas (enfermedades cardiovasculares, respiratorias y renales crónicas, diabetes mellitus, alcoholismo, daño hepático crónico, asplenia), e inmunocomprometidos.

Vacuna antiinfluenza. La eficacia de la vacuna depende de múltiples factores, entre otros, la coincidencia del virus presente en la comunidad con el incluido en la vacuna, factores ambientales y factores del huésped. Se estima que en el adulto sano, la eficacia varía entre 70 y $90 \%$; en sujetos mayores de 60 años, no institucionalizados, es de $58 \% \mathrm{y}$, en adultos mayores residentes en centros geriátricos, disminuye a 30\%-40\%. En un metaanálisis que examinó veinte estudios de cohorte $^{34}$ se demostró que la eficacia variaba de acuerdo a los resultados que querían obtenerse: a) la prevención de la enfermedad respiratoria fue de $56 \%$ (IC $95 \%$ : $39 \%$ a $68 \%$ ), b) la neumonía fue de $53 \%$ (IC 95\%: $35 \%$ a $66 \%)$, c) la admisión al hospital fue de $50 \%$ (IC 95\%: $28 \%$ a $65 \%$ ) y, d) el riesgo de muerte fue de $68 \%$ (IC $95 \%$ : $56 \%$ a $76 \%$ ).

Se recomienda vacunar anualmente contra la influenza a los adultos mayores ( $\geq 65$ años), portadores de enfermedades crónicas de cualquier edad (enfermedades cardiovasculares, respiratorias, renal y hepática crónicas, diabetes mellitus), embarazadas con más de 12 semanas de gestación y pacientes inmunocomprometidos.

7. Fine MJ, Smith Ma, Carson CA, Mutha SS, Sankey SS, WeISSFeld LA et AL. Prognosis and outcomes of patients with community-acquired pneumonia. A meta-analysis. JAMA 1996; 275: 134-41.

8. Saldías F, O’Brien A, Gederini A, Farías G, Díaz A. Neumonía adquirida en la comunidad en el anciano inmunocompetente que requiere hospitalización. Cuadro clínico, factores pronósticos y tratamiento. Arch Bronconeumol 2003; 39: 333-40.

9. FeduLn AJ, SwinBurne AJ. Relationship of patient age to clinical features and outcome for inhospital treatment of pneumonia. J Geront 1985; 40: 29-33.

10. Koivula I, Sten M, Makeia PH. Risk factors for pneumonia in the elderly. Am J Med 1994; 96: 313-20.

11. Janssens JP, Pache JC, Nicod LP. Physiological changes in respiratory function associated with ageing. Eur Respir J 1999; 13: 197-205. 
12. Gyetko MR, Toews GB. Immunology of the aging lung. Clin Chest Med 1993; 14: 379-91.

13. Logroscino CD, Penza O, Locicero S, Losito G, NaRdini S, Bertoli L et al. Community-acquired pneumonia in adults: a multicentric observational AIPO study. Monaldi Arch Chest Dis 1999; 54: 1117.

14. Fernández-Sabe N, Carratala J, Ronson B, Dorca J, Verdaguer R, ManResa F et al. Community-acquired pneumonia in very elderly patients: causative organisms, clinical characteristics, and outcomes. Medicine 2003; 82: 159-69.

15. Saldías F, Mardonez JM, Marchesse M, Viviani $P$, FARÍAS G, DíAZ A. Neumonía adquirida en la comunidad en el adulto hospitalizado. Cuadro clínico y factores pronósticos. Rev Méd Chile 2002; 130: 1373-82.

16. Díaz A, Fuentes G, Couble B, Uribe R, Mercado G, SozA A ET AL. Etiología de la neumonía adquirida en la comunidad en adultos hospitalizados en Santiago, Chile: implicancias para las guías clínicas. Rev Chil Enf Respir 2005; 21: 23-32.

17. Johnson JC, JaYadevapPa R, Baccash PD, TaYLor L Nonspecific presentation of pneumonia in hospitalized older people: age effect or dementia? J Am Geriatr Soc 2000; 48: 1316-20.

18. Fein AM, Feinsilver SH, Niederman MS. Atypical manifestations of pneumonia in the elderly. Clin Chest Med 1991; 12: 319-36.

19. Conte HA, Chen YT, Mehal W, Scinto JD, QuaglaRELO VJ. A prognostic rule for elderly patients admitted with community-acquired pneumonia. Am J Med 1999; 106: 20-8.

20. Fine MJ, Auble TE, Yealy DM, Hanusa BH, WeissFELD LA, Singer DE et aL. A prediction rule to identify low-risk patients with community-acquired pneumonia. N Engl J Med 1997; 336: 243-50.

21. Saldías F, Farías G, Vilarroel L, Valdivia G, Mardonez JM, Díaz A. Diseño de un índice pronóstico clínico para el manejo de la neumonía del adulto adquirida en la comunidad. Rev Méd Chile 2004; 132: 1037-46.

22. Petitpretz P, Arvis P, Marel M, Moita J, Urueta J. Oral moxifloxacin vs high-dosage amoxicillin in the treatment of mild-to-moderate, communityacquired, suspected pneumococcal pneumonia in adults. Chest 2001; 119: 185-95.

23. Jardim JR, Rico G, De La Roza C, Obispo E, Urueta J, WolfF M ET AL. A comparison of moxifloxacin and amoxicillin in the treatment of community-acqui- red pneumonia in Latin America: results of a multicenter clinical trial. Arch Bronconeumol 2003; 39: 387-93.

24. Chan R, Hemeryck L, O'regan M, Clancy L, Feely J. Oral versus intravenous antibiotics for community acquired lower respiratory tract infection in a general hospital: open, randomized controlled trial. BMJ 1995; 310: 1360-2.

25. Rovira E, Martínez-Moragon E, Belda A, Gonzalvo F, RipoLes F, PASCUAL JM. Treatment of communityacquired pneumonia in outpatients: randomized study of clarithromycin alone versus clarithromycin and cefuroxime. Respiration 1999; 66: 413-8.

26. BJERRE LM, VERHEIJ TJ, Kochen MM. Antibiotics for community acquired pneumonia in adult outpatients. Cochrane Database Syst Rev 2004; (2): CD 002109.

27. MiLs GD, Oehiey MR, Arrol B. Effectiveness of $\beta$ lactam antibiotics compared with antibiotics active against atypical pathogens in non-severe community acquired pneumonia: meta-analysis. BMJ 2005; 330 (7489): 456.

28. Saldías F, Flores LJ, Torres C, García P, Díaz A. Susceptibilidad a antimicrobianos de Streptococcus pneumoniae en población infantil y adulta de Santiago. Período 1997-2003. Rev Méd Chile 2005; 133: 42-9.

29. Ewig S, Ruiz M, Torres A, Marco F, Martínez JA, Sánchez $M$ ET al. Pneumonia acquired in the community through drug-resistant Streptococcus pneumoniae. Am J Respir Crit Care Med 1999; 159: $1835-42$.

30. Feikin DR, Schuchat A, Kolczak M, Barret NL, HARRISON LH, LEFKOWITZ L ET AL. Mortality from invasive pneumococcal pneumonia in the era of antibiotic resistance, 1995-1997. Am J Public Health 2000; 90: 223-9.

31. BTS Guidelines for the management of community acquired pneumonia in adults - 2004 update.

32. BRITton S, BejSTEDt M, Vedin L Chest physiotherapy in primary pneumonia. Br Med J 1985; 290: 1703-4.

33. Butler JC, Breiman RF, Campbell JF, Lipman hB, Broome CV, Fackiam RR. Pneumococcal polysaccharide vaccine efficacy. An evaluation of current recommendations. JAMA 1993; 270: 1826-31.

34. Gross PA, Hermogenes AW, Sacks HS, Lau J, LEVANDOWSKI RA. The efficacy of influenza vaccine in elderly persons. A meta-analysis and review of the literature. Ann Intern Med 1995; 123: 518-27. 